\title{
Actividad física y nutrición en la educación médica
}

\author{
Physical activity and nutrition in medical education \\ Atividade física e nutrição na educação médica
}

\author{
Gustavo Tovar Mojica1* , Erika Viviana Ladino Marín
}

Recibido: 31 de mayo de 2019. Aceptado para publicación: 5 de agosto de 2019

https://doi.org/10.35454/rncm.v2n2.005

\begin{abstract}
Resumen
Las enfermedades no transmisibles (ENT) son la principal causa de muerte en todo el mundo (70\%). En Colombia mueren por ENT 178.000 personas al año, $73 \%$ de todas las muertes, debido, en gran medida, a las malas prácticas en los estilos de vida, especialmente inactividad física, sobrealimentación y tabaquismo. En Colombia, el exceso de peso ha aumentado en la población adulta de 18 a 65 años $(56,5 \%)$ mientras que el porcentaje de personas adultas que cumplen las recomendaciones de actividad física (51,3\%) ha venido en descenso. El médico, y en general el personal de salud, tienen un papel fundamental para ayudar a los pacientes y sus familias en la construcción de un estilo de vida saludable. Los temas de actividad física y nutrición deben ser parte obligatoria de los currículos de las carreras de salud. Para el tema de nutrición se recomienda centrar la instrucción en cuatro ejes: nutrición básica, diagnóstico clínico nutricional, intervención nutricional y tratamiento dietario de la enfermedad, y en el tema de actividad física se recomienda que la intervención se enfoque en cuatro aspectos: evaluación de la actividad física y de la condición física, prescripción e implementación del ejercicio, consejería y estrategias de cambio comportamental y salud del personal médico. Se muestra la experiencia de los autores con el programa de Medicina del Deporte de la Escuela de Medicina y Ciencias de la Salud de la Universidad del Rosario.
\end{abstract}

Palabras clave: ejercicio, salud pública, curriculum, Enfermedades no transmisibles.

\section{Summary}

Noncommunicable diseases (NCDs) are the leading cause of death worldwide (70\%). In Colombia, 178,000 people per year $(73 \%)$ die from NCDs. Bad practices in lifestyles, especially physical inactivity, overfeeding and smoking leading to NCDs. In Colombia, overweight has increased in the entire population: $56.5 \%$ in adults aged 18 to 65 years and the percentage of people who meet the physical activity recommendations is only $51.3 \%$ in adults. Physicians and in general health professionals have a fundamental role to help patients and their families in the construction of a healthy lifestyle. The subject of physical activity and nutrition must be compulsory part of the curricula of health careers. For nutrition, instruction is recommended centered on 4 axes: basic nutrition, nutritional assessment, nutritional intervention and dietary treatment of disease. In physical activity it is recommended that the intervention focuses on four aspects: physical activity and fitness cardiorespiratory assessment exercise prescription, counseling and behavioral change strategies and health of medical personnel. The experience of the authors is shown with the sports medicine and exercise program of the School of Medicine and Health Sciences of the Universidad del Rosario.

Key words: Exercise; Curriculum; Public Health; Noncommunicable diseases.

\section{Resumo}

As doenças não transmsiveis (DNTs) são principal causa de morte no mundo (70\%). Na Colômbia morrem 178.000 pessoas por ano, $73 \%$ de todas as mortes, devido, em grande parte, a más práticas nos estilos de vida, especialmente inatividade física, sobrealimentação e tabaco. Na Colômbia, o excesso de peso aumentou na população adulta de 18 a 65 anos (56,5\%) e a percentagem de adultos que cumprem as recomendações de atividade física $(51,3 \%)$ vem diminuiendo. O médico, e em geral a equipe de saúde, tem um papel fundamental para ajudar os pacientes e suas famílias na construção de um estilo de vida saudáveil. Os temas da atividade física e nutrição devem ser uma parte obrigatória nos currículos dos cursos de saúde. Para a questão da nutrição, recomenda-se a instrução centrada em quatro eixos: nutrição básica, diagnóstico nutricional clínico, intervenção nutricional e tratamento dietético da doença, no tema da atividade física recomenda-se que a intervenção seja focada em quatro aspetos: avaliação do atividade e condição física, prescrição e implementação de estratégias de exercício, aconselhamento e mudança comportamental, saúde da equipa médica. É relatada la experiência dos autores com o programa de medicina desportiva da Faculdade de Medicina e Ciências da Saúde da Universidad del Rosario.

Palavras-chave: exercício, currículo, saúde pública, doenças não transmissíveis.
1 Medicina del Deporte, grupo Mente y Cuerpo. Escuela de Medicina y Ciencias de la Salud Universidad del Rosario, Bogotá, D.C, Colombia.
Correspondencia: *Gustavo Tovar Mojica gustavo.tovar@urosario.edu.co 


\section{INTRODUCCIÓN}

Las enfermedades no transmisibles (ENT), las enfermedades cardiovasculares (ECV), el cáncer, la diabetes y las enfermedades respiratorias crónicas (ERC), son la principal causa de muerte en todo el mundo (70 \%). En Colombia mueren por ENT 178.000 personas al año, que corresponde a $73 \%$ de todas las muertes ${ }^{(1)}$, debido, en gran medida, a las malas prácticas en los estilos de vida y especialmente a la inactividad física, sobrealimentación y tabaquismo ${ }^{(2)}$.

La Organización Mundial de la Salud (OMS) aconseja desde 2004 trabajar sobre alimentación saludable, actividad física y control del tabaco para lograr la disminución de la prevalencia de enfermedades crónicas. En el reporte global sobre enfermedades no transmisibles publicado en 2014, cuatro de sus nueve objetivos involucran directamente a la nutrición y a la actividad física; ${ }^{(3)}$ sin embargo, se está perdiendo la batalla. Las metas fijadas por la OMS para el control de los cuatro factores de riesgo comunes y modificables de la ENT (tabaco, dietas malsanas, falta de actividad física y consumo nocivo de alcohol) para el año 2025 no están siendo suficientes ${ }^{(1)}$. La inactividad física es el factor de riesgo más prevalente de las enfermedades no trasmisibles ${ }^{(4)}$. En Colombia ha venido aumentando el exceso de peso en la población: $56,5 \%$ en adultos de 18 a 65 años (5,3 puntos porcentuales de aumento de 2010 a 2015), 17,9\% en adolescentes, $24,4 \%$ en escolares y ha venido bajando el porcentaje de personas que cumplen las recomendaciones de actividad física en $51,3 \%$ en adultos ${ }^{(5)}$.

El médico, y en general el personal de salud, tienen un papel fundamental para ayudar a los pacientes y sus familias en la construcción de un estilo de vida saludable sostenible en el tiempo ${ }^{(6)}$, por lo que se vuelve una necesidad del siglo XXI la generación de capacidades que les permitan recomendar la actividad física, medir el fitness cardiorrespiratorio y hacer un correcto diagnóstico del estado nutricional que los lleve a detectar los sujetos que necesitan una atención especializada por equipos interdisciplinarios, con especialistas en medicina del deporte y en nutrición ${ }^{(6,7)}$. El fitness cardiorrespiratorio es un predictor de mortalidad tan fuerte como la diabetes, el tabaco, la hipertensión arterial o la hipercolesterolemia. Por cada Metabolic Equivalent of Task (MET) de incremento en la condición cardiovascular se aumenta en forma considerable la sobrevida ( $10 \%$ a $30 \%$ ), si se logra que el personal de salud detecte los individuos con muy baja condición física y que el sistema de salud intervenga para que mejoren su con- dición (llevarlos de 4 METs a 5 o 6 METs), se lograría disminuir a la mitad su riesgo de morir ${ }^{(8)}$.

La actividad física habitual es un pilar fundamental de la promoción de salud y la prevención de enfermedad, impulsa otros hábitos saludables como el dejar de fumar, mejorar la alimentación y disminuir el consumo de alcohol(9); impacta en forma importante la prevención, el manejo y la rehabilitación de 8 condiciones crónicas: falla cardiaca, enfermedad coronaria, dislipidemia, hipertensión, resistencia a la insulina, claudicación intermitente, obesidad y diabetes tipo $2^{(10)}$, además de intervenir la patogénesis de la osteoporosis, los síntomas de la enfermedad pulmonar obstructiva crónica (EPOC), depresión, fibromialgia y osteoartritis. Se han comprobado sus beneficios en asma, síndrome de fatiga crónica, artritis reumatoide y en algunas formas de cáncer (en especial seno y colon), adicionalmente mejora el equilibrio, la función cognitiva, la expectativa y la calidad de vida, y se conoce su papel en la disminución en la aparición de demencia, caídas y sarcopenia, además de reducir los costos de la atención médica ${ }^{(11-13)}$.

El consumo de frutas y verduras está relacionado por sí solo como un factor protector en la aparición de enfermedades crónicas como el cáncer, la enfermedad cardiaca, los accidentes cerebro vasculares, e inclusive reduce el riesgo de cataratas, diverticulosis, enfermedad pulmonar obstructiva crónica e hipertensión ${ }^{(14)}$. Las alteraciones en la alimentación y la inactividad física son los mayores contribuyentes de la obesidad, diabetes tipo 2, el cáncer y la enfermedad cardiovascular ${ }^{(15)}$.

\section{IMPORTANCIA DE LA ACTIVIDAD FÍSICA Y LA NUTRICIÓN EN LA EDUCACIÓN MÉDICA}

La educación médica en los últimos 40 años ha venido incrementando la formación relacionada con Nutrición y Actividad Física, aunque en forma lenta. En 1974 se realizó una encuesta que mostró algo de instrucción en este tema en $16 \%$ de las escuelas de medicina en los Estados Unidos ${ }^{(16,17)}$. Otra encuesta realizada a decanos de medicina en 2001 mostró que $61 \%$ considera que la instrucción sobre actividad física es un tema importante, pero solo $6 \%$ de las escuelas encuestadas tenían un curso formal de prescripción de ejercicio ${ }^{(16,18)}$. En nutrición pasa algo similar: una encuesta realizada en 1985 encontró que solo $20 \%$ de las escuelas de medicina tenía un curso obligatorio en nutrición ${ }^{(19)}$, y la Asociación Americana de Colegios Médicos demostró que más de la mitad de los estudiantes $(51,5 \%)$ no tuvo una adecuada instrucción en temas de nutrición ${ }^{(20,21)}$. 
En 2014, autoridades de nutrición se alertaron al encontrar que solo $27 \%$ de las escuelas de medicina dictaban algún curso formal de nutrición y su promedio era de 20 horas $^{(16,22)}$.

De acuerdo con los inminentes requerimientos en salud pública, múltiples autoridades mundiales han emitido recomendaciones urgentes de implementación para lograr un mayor énfasis en la enseñanza de la nutrición y actividad física en todos los niveles de la educación médica. "The Global Advocacy for Physical Activity (GAPA) de la "International Society for Physical Activity and Health (ISPAH)" en "7 investments that work" hacen énfasis en la necesidad de darle fuerza a los comportamientos saludables en todos los sistemas de salud ${ }^{(23)}$. El Bipartisan Policy Center en Washington, D.C., en 2012, mencionó: "El entrenamiento en nutrición y actividad física debe ser incorporado en todas las fases de la educación médica" escuelas de medicina, programas de residencia y educación continuada ${ }^{(24)}$, confirmando la necesidad de desarrollar e implementar un currículo estándar sobre nutrición y actividad física en alianza con el Colegio Americano de Medicina del Deporte ${ }^{(25)}$. En 2007, el Colegio Americano de Medicina del Deporte creó la iniciativa "Exercise is medicine" para ayudar a mejorar la salud y el bienestar a través de la medición de la actividad física como un signo vital y ayudar a que médicos y personal de salud aprendan a prescribir actividad física ${ }^{(26)}$. De igual manera, en 2013 el Plan Nacional de Actividad Física de los Estados Unidos propuso incluir la enseñanza en actividad física en todos los niveles de educación de los profesionales de salud ${ }^{(27)}$. En la escuela de medicina Greenville de la Universidad de Carolina del Sur, en el año 2012, decidieron incorporar lo aconsejado por Exercise is medicine, a lo largo de los 4 años de su plan de estudios médicos, y organizaron el primer "Think Tank" en el año 2013, en conjunto con el Instituto de Medicina del Estilo de Vida (Institute of Lifestyle Medicine), el Joslin Diabetes Center y la Universidad de Harvard, con la idea de estimular la adopción de este tipo de currículos en otras escuelas de medicina. Este Think Tank tuvo representantes del Colegio Americano de Medicina del Deporte, del Instituto Nacional de Salud de USA, Asociación Médica Americana, Junta Nacional de Examinadores Médicos, Bipartisan Policy Center, entre otras partes interesadas y se propuso la creación de un currículo en Medicina del Estilo de Vida "Lifestyle Medicine"(16).

En Inglaterra durante los años 2017 y 2018 se creó una comisión formada por Salud Pública (Public Health
England) y por "Deporte inglés" (Sport England) como parte de la estrategia conjunta "Moving Healthcare Professionals Programme" la cual visitó 16 de las 17 escuelas inglesas de medicina y contactó a 83 escuelas de salud para conocer la forma como la actividad física estaba involucrada en los currículos, lo que resulta en un marco para su implementación ${ }^{(28)}$.

Las autoridades mencionadas recomiendan que los contenidos de actividad física y nutrición deben hacer parte obligatoria de los currículos de formación para todos los estudiantes. Su enseñanza debe estar enmarcada dentro de conceptos de epidemiología y atención primaria en salud, para que puedan abordar al paciente de una forma holística y se entiendan los diferentes determinantes de la salud y la enfermedad. Los temas de actividad física y nutrición, en forma independiente o agrupados en medicina de los estilos de vida, deben hacer parte de todos los programas de las carreras de salud a lo largo de su plan de estudios tanto en pregrado como en posgrado, además se deben generar cursos de educación continua para los profesionales que trabajan en áreas de atención primaria y movilizar a los estudiantes y profesionales de áreas de la salud a ser más activos, dada la constante asociación positiva entre sus hábitos personales y la posibilidad de recomendar la actividad física a sus pacientes. Los estudiantes que han sido expuestos a currículos que favorecen los estilos de vida saludable tienden a tener mejores hábitos y a generar mayor prescripción de ejercicio en sus pacientes ${ }^{(29-31)}$.

Los contenidos del currículo de estilos de vida proponen foco en cuatro puntos principales: actividad física, nutrición, auto cuidado y cambio comportamental. Para nutrición recomiendan centrar la instrucción en cuatro ejes: nutrición básica, diagnóstico clínico nutricional, intervención nutricional y tratamiento dietario de la enfermedad, mientras que para actividad física se propone enfocar la intervención en cuatro aspectos: evaluación de la actividad física y de la condición física, prescripción e implementación del ejercicio, consejería y estrategias de cambio comportamental y salud del personal médico ${ }^{(16,21,24,32)}$.

\section{AVANCES DE COLOMBIA EN EL CURRÍCULO}

En Colombia, el Plan Decenal de Salud Pública (PDSP) 2012 - 2021 exhorta a trabajar en la promoción de la salud y en la prevención de la enfermedad y define ocho dimensiones prioritarias, una de ellas denominada "dimensión de vida saludable y condiciones no transmisibles" busca identificar e intervenir 
los factores de riesgo. Incluye tres metas específicas de nutrición: reducción del consumo de sal, incremento del consumo de frutas y de verduras, y cinco metas para la actividad física que buscan su incremento en el sistema educativo, en los diferentes grupos de edad y el estímulo al transporte activo ${ }^{(33)}$.

En el año 2007 se realizaron mediciones en 24 facultades de medicina del país con una adaptación del cuestionario de "Healthy Doc = Healthy Patient", dentro de un estudio llamado "Prevención de Enfermedades Crónicas: una prioridad en la formación médica”, liderado por el doctor Jhon Duperly - Universidad de los Andes. El estudio revisa la asociación entre cuatro hábitos (actividad física, nutrición, tabaquismo y consumo de alcohol), las actitudes frente a la consejería, y los conocimientos de los estudiantes de primero y quinto año de medicina. Contempla una segunda y tercera fase encaminadas a generar estrategias de intervención en hábitos saludables y actitudes hacia la consejería preventiva. Posteriormente se realizaron varios encuentros nacionales con representantes de cada facultad para socializar una gran cantidad de iniciativas que surgieron en cada facultad encuestada, la mayor parte de ellas de tipo extracurricular y varias inmersas en los currículos que lograron posicionar el tema en cada universidad ${ }^{(29,34,35)}$.

A continuación, mostramos nuestra experiencia con el programa de medicina del deporte en el currículo de medicina de la Escuela de Medicina y Ciencias de la Salud: en Bogotá, en 1999 la Universidad del Rosario creó un espacio voluntario de actividad física y ejercicio para los estudiantes y en 2001 se incorporó como un espacio obligatorio de la cátedra de medicina interna, con una representación de $10 \%$ de la nota final. En el año 2007 se consolidó como una materia obligatoria con un crédito y una intensidad horaria de tres horas a la semana, con el objetivo de mejorar las condiciones de salud mediante el fomento de la actividad física y formar al estudiante, de manera práctica, en la metodología del entrenamiento físico y que vivencie sus beneficios. El $70 \%$ de las clases contenía ejercicio físico grupal dirigido y $30 \%$ de contenido teórico, con el fin de transmitir la influencia de la actividad física sobre las diferentes patologías, enseñar los beneficios fisiológicos como producto de una adaptación del cuerpo hacia las cargas de ejercicio, y herramientas generales para la prescripción del ejercicio.

Después de analizar siete cohortes que recibieron la clase, se evidencia un incremento en las condiciones de salud de los estudiantes ${ }^{(30)}$. En otro análisis que compara estudiantes de medicina de la Universidad del
Rosario con la muestra nacional del estudio realizado por la Universidad de Los Andes se evidencian algunas diferencias en actividad física y ejercicio (mayores niveles en estudiantes de la Universidad del Rosario), que podrían corresponder a un impacto del programa instaurado ${ }^{(31)}$.

En el año 2013, el currículo del programa de Medicina de la Universidad del Rosario se reformó integrando las ciencias básicas, biomédicas, clínicas, socio humanísticas y de salud de poblaciones, a través de un método de enseñanza aprendizaje basado en Actividades Integradoras del Aprendizaje por Sistemas (AIAS) $)^{(36)}$, permitiendo al tema de actividad física y ejercicio integrarse al currículo en diferentes momentos, desde el segundo hasta el sexto semestre, en forma de clases prácticas y teóricas en la mayoría de las nuevas AIAS (Tabla 1).

En las clases teóricas (9 horas - $35 \%$ ) se vincula el papel de la actividad física y el ejercicio en el tema que se está revisando y se explican sus beneficios desde la fisiología, se brindan herramientas básicas de prescripción del ejercicio y se incluye siempre una pausa activa en la mitad de la exposición teórica. En las clases prácticas ( 17 horas $-65 \%$ ), el estudiante realiza actividad física de forma lúdica, aprende la metodología del ejercicio y se enseña sobre su relación con el tema del AIAS correspondiente. Un objetivo fundamental es generar conciencia sobre sus propios hábitos, por lo que incluye la entrega de un informe individual con retroalimentación de sus resultados y recomendaciones de mejora. En cuarto semestre se imparte un curso voluntario de hábitos de vida saludable en donde la escuela de medicina les brinda monitores cardiacos para medir la actividad física diaria y frecuencia cardiaca (Polar A 300), monitorizar los comportamientos sedentarios o activos y se brinda información sobre hábitos saludables durante un periodo de 14 semanas, lo que genera líderes naturales en el área de medicina del deporte que colaboran en mediciones físicas, manejo de los dispositivos y mejoran su propia experiencia con hábitos de vida sana.

Actualmente, por iniciativa de la decanatura de la escuela de medicina y ciencias de la salud de la Universidad del Rosario, se crea el grupo "Mente y Cuerpo" de trabajo interdisciplinario en convenio con la Fundación Cardioinfantil, teniendo en cuenta cuatro componentes: autocuidado, mente y cuerpo en el currículo, formación docente y educación al paciente y a su cuidador; con la investigación como centro, encaminados a fortalecer la formación del médico desde las perspectivas académicas y sociales. 
Tabla 1. Medicina del deporte en el currículo del programa de medicina Universidad del Rosario año 2019.

\begin{tabular}{|c|c|c|c|}
\hline SEM & AIAS & Módulo/caso & Sesión \\
\hline II Semestre & $\begin{array}{l}\text { AIAS Hemato/Onco e Infectología } \\
\text { AIAS Líquidos y Electrolitos } \\
\text { AIAS Líquidos y Electrolitos }\end{array}$ & $\begin{array}{l}\text { Cáncer de seno } \\
\text { Hiponatremia } \\
\text { Acidosis metabólica }\end{array}$ & $\begin{array}{l}\text { Pruebas físicas para valorar el estado de salud II } \\
\text { Introducción a la medicina del deporte } \\
\text { Pruebas físicas para valorar el estado de salud I }\end{array}$ \\
\hline III Semestre & $\begin{array}{l}\text { AIAS Medicina del } \\
\text { comportamiento } \\
\text { AIAS Medicina del } \\
\text { comportamiento } \\
\text { AIAS Medicina del } \\
\text { comportamiento } \\
\text { AIAS Sistema Nervioso } \\
\text { AIAS Sistema Nervioso } \\
\text { AIAS Sistema Nervioso } \\
\text { AIAS Sistema Nervioso }\end{array}$ & $\begin{array}{l}\text { Afecto } \\
\text { Trastornos del } \\
\text { pensamiento } \\
\text { Agresión y violencia } \\
\text { Síndrome convulsivo } \\
\text { Ataque cerebrovascular } \\
\text { Trastornos del } \\
\text { movimiento } \\
\text { Cefalea }\end{array}$ & $\begin{array}{l}\text { Medicina del deporte (depresión y ejercicio) } \\
\text { Medicina del deporte (cerebro y ejercicio) } \\
\text { Medicina del deporte (autorregulación y actividad } \\
\text { física) } \\
\text { Epilepsia y ejercicio. Ejercicios de propiocepción } \\
\text { Fisiología cardiovascular y ejercicio. Prescripción de } \\
\text { ejercicio en paciente con ACV } \\
\text { Parkinson y ejercicio. Ejercicios de marcha } \\
\text { Cefalea tensional. Ejercicios de estiramiento y } \\
\text { tonificación cervical }\end{array}$ \\
\hline IV Semestre & $\begin{array}{l}\text { AIAS Cardiovascular } \\
\text { AIAS Cardiovascular } \\
\text { AIAS Cardiovascular } \\
\text { AIAS Cardiovascular } \\
\text { AIAS Endocrinología } \\
\text { AIAS-Músculo esquelético } \\
\text { AIAS-Músculo esquelético }\end{array}$ & $\begin{array}{l}\text { Hipertensión } \\
\text { Palpitaciones } \\
\text { Paro cardiorrespiratorio } \\
\text { Obesidad } \\
\text { Diabetes } \\
\text { Lumbalgia } \\
\text { Trauma }\end{array}$ & $\begin{array}{l}\text { Hipertensión y ejercicio. Ejercicios de intensidad } \\
\text { moderada } \\
\text { Ejercicios de alta intensidad, HIIT } \\
\text { Corazón y ejercicio. Frecuencia cardiaca y ejercicio } \\
\text { Obesidad, ejercicio y cálculos de calorías } \\
\text { Prescripción de ejercicio y beneficios para paciente con } \\
\text { Diabetes } \\
\text { Ejercicios para prevenir el dolor de espalda y tonificar } \\
\text { CORE } \\
\text { Medicina del deporte: prevención de lesiones del } \\
\text { ejercicio y medición de la intensidad del ejercicio }\end{array}$ \\
\hline V Semestre & $\begin{array}{l}\text { AIAS-Gastroenterología } \\
\text { AIAS-Respiratorio } \\
\text { AIAS-Respiratorio }\end{array}$ & $\begin{array}{l}\text { Vómito y náuseas } \\
\text { Asma } \\
\text { EPOC }\end{array}$ & $\begin{array}{l}\text { Cáncer de colon y ejercicio. Ejercicios de } \\
\text { fortalecimiento abdominal } \\
\text { Asma y ejercicio. Ejercicios de respiración y } \\
\text { fortalecimiento de músculos respiratorios } \\
\text { Cambios respiratorios con ejercicio, EPOC y } \\
\text { broncoespasmo inducido por ejercicio }\end{array}$ \\
\hline VI Semestre & $\begin{array}{l}\text { AIAS-Dermatología } \\
\text { AIAS-Envejecimiento } \\
\text { AIAS-Renal } \\
\text { AIAS-Renal } \\
\text { AIAS-Reproductivo }\end{array}$ & $\begin{array}{l}\text { Neoplasia de piel } \\
\text { Demencia } \\
\text { Enfermedad glomerular } \\
\text { Enfermedad glomerular } \\
\text { Aborto }\end{array}$ & $\begin{array}{l}\text { Protección solar y ejercicio. Ejercicios de } \\
\text { fortalecimiento miembros inferiores } \\
\text { Envejecimiento y ejercicio } \\
\text { IRC y ejercicio. Ejercicios para mejorar la calidad de vida } \\
\text { de pacientes IRC } \\
\text { Hidratación y cuidado del riñón con el ejercicio } \\
\text { Triada de la mujer atleta. Ejercicios para tonificar. }\end{array}$ \\
\hline
\end{tabular}

AIAS: Actividades Integradoras del Aprendizaje por Sistemas

\section{EL PRÓXIMO PASO}

Las ENT y su gran carga de enfermedad hacen necesario que cambie la forma de abordar los hábitos saludables en el sistema de salud. Las estadísticas del sector siempre actualizan prevalencias de enfermedad y no han comenzado a medir indicadores positivos de salud que direccionen mejor las acciones de promoción y prevención $^{(37)}$. Un indicador positivo es el que evalúa el cumplimiento de los siguientes tres criterios: actividad física para su grupo de edad (recomendaciones OMS), no fumar y peso saludable para la edad. Permite identificar la población con "indicador saludable" y trabajar para lograr su incremento, como objetivo compartido de todos los actores del Sistema de Seguridad Social, a través de acciones de promoción de la actividad física, 
alimentación saludable y la batalla contra el tabaquismo $\mathrm{y}$ otras drogas de abuso. Estas acciones pueden llegar a generar incentivos (como la disminución en costos de planes de salud), estimular al sistema de salud para que se enfoque principalmente en actividades de prevención y promoción ${ }^{(6)}$.

La evaluación de la condición física, ya sea por la medición del fitness cardiovascular o al menos por medio de preguntas en la historia clínica como un signo vital, no ha sido considerada con la suficiente seriedad por las autoridades de salud. Los médicos especialistas en medicina del deporte y los nutricionistas deben brindar la educación y los métodos necesarios para que el personal de salud pueda evaluar en los pacientes el nivel de actividad física, su capacidad funcional y su estado nutricional, con el fin de brindar una consejería adecuada y remitir a un especialista cuando sea necesario. El médico especialista en medicina del deporte y el ejercicio debe colaborar con la formación y dirección de programas interdisciplinarios de prevención y promoción de la salud que incluyan educadores físicos, con el fin de incrementar el uso del ejercicio como una modalidad terapéutica, ayudar a los pacientes a incrementar su actividad, establecer y mantener una relación adecuada entre la industria del fitness con el cuidado médico tanto en el nivel individual como en el comunitario.

La tendencia en el desarrollo y uso de la tecnología permite en la actualidad obtener datos de objetos o prendas con sensores de actividad física (wearables), inclusive en nuestros teléfonos móviles, como herramienta útil para la vigilancia del comportamiento activo, al lograr que la data se incorpore de forma automática a la historia clínica de cada sujeto y pueda ser analizada y utilizada por el personal de salud ${ }^{(38)}$.

En conclusión, la generación de conciencia y apropiación de conocimientos acerca de la importancia de la actividad física y nutrición saludable desde los profesionales de la salud en formación, permitirá lograr impacto en la promoción de la salud y la prevención de las enfermedades no transmisibles en la población general. Finalmente, como dice el Plan Decenal de Salud Pública, la salud debe estar inmersa en las políticas de todos los sectores gubernamentales y no gubernamentales, que deberán aportar lo que corresponda desde su competencia para favorecer comportamientos activos, nutrición adecuada y para el logro del objetivo final de una mejor salud y bienestar.

\section{Financiación}

El presente artículo no tuvo financiación.

\section{Conflicto de intereses}

Los autores declaran no tener conflicto de intereses.

\section{Declaración de autoría}

Los autores: Gustavo Tovar y Erika Viviana Ladino declaramos haber participado en la concepción y realización de este trabajo, haber participado en la redacción del texto y en sus revisiones, y aprobado la versión final.

\section{Referencias bibliográficas}

1. Organización Mundial de la Salud. Monitoreo de avances en materia de las enfermedades no transmisibles 2017. Switzerland.2017. Available from: https://apps.who.int/iris/ handle/10665/259806 [consultado 6 de junio 2019].

2. Chopra M, Galbraith S, Darnton-Hill I. A global response to a global problem: the epidemic of overnutrition. Bull World Health Organ. 2002;80(12):952-8.

3. Organization WH. Global status report on noncommunicable diseases. Switzerlanul2014. Available from: www.who.int/ ncd. [consultado 6 de Junio 2019]

4. Blair SN. Physical inactivity: the biggest public health problem of the 21st century. Br J Sports Med. 2009;43(1):1-2.

5. Instituto Colombiano de Bienestar Familiar I, Minsalud, Prosperidad Social, Instituto Nacional de Salud, Universidad Nacional de Colombia. Encuesta nacional de la situación nutricional en Colombia (ENSIN) 2015. 2015.

6. Joy E, Blair SN, McBride P, Sallis R. Physical activity counselling in sports medicine: a call to action. British Journal of Sports Medicine. 2013;47(1):49-53.

7. Weiler R, Chew S, Coombs N, Hamer M, Stamatakis E. Physical activity education in the undergraduate curricula of all UK medical schools: are tomorrow's doctors equipped to follow clinical guidelines? $\mathrm{Br} \mathrm{J}$ Sports Med. 2012;46(14):1024-6.

8. Ross R, Blair SN, Arena R, Church TS, Després JP, Franklin $\mathrm{BA}$, et al. Importance of Assessing Cardiorespiratory Fitness in Clinical Practice: A Case for Fitness as a Clinical Vital Sign: A Scientific Statement From the American Heart Association. Circulation. 2016;134(24):e653-e99.

9. Pate RR, Heath GW, Dowda M, Trost SG. Associations between physical activity and other health behaviors in a representative sample of US adolescents. Am J Public Health. 1996;86(11):1577-81.

10. Pedersen BK, Saltin B. Evidence for prescribing exercise as therapy in chronic disease. Scand J Med Sci Sports. 2006; 16 Suppl 1:3-63.

11. Lee IM, Shiroma EJ, Lobelo F, Puska P, Blair SN, Katzmarzyk $\mathrm{PT}$, et al. Effect of physical inactivity on major non-communicable diseases worldwide: an analysis of burden of disease and life expectancy. Lancet. 2012;380(9838):219-29. 
12. Meltz J, Heffernan A. The exercise cure: a doctor's all-natural, no-pill prescription for better health \& longer life. Emmaus, editor. Pennsylvania 2013. p. 298.

13. Cardinal BJ, Park EA, Kim M, Cardinal MK. If Exercise is Medicine, Where is Exercise in Medicine? Review of U.S. Medical Education Curricula for Physical Activity-Related Content. J Phys Act Health. 2015;12(9):1336-43.

14. Van Duyn MA, Pivonka E. Overview of the health benefits of fruit and vegetable consumption for the dietetics professional: selected literature. J Am Diet Assoc. 2000;100(12):1511-21.

15. Organization $W H$. Preventing chronic diseases a vital investment: WHO global report. Switzerland 2005. Available from: www.who.int/chp/chronic_disease_report/en/.[consultado 6 de Junio 2019]

16. Phillips E, Pojednic R, Polak R, Bush J, Trilk J. Including lifestyle medicine in undergraduate medical curricula. Med Educ Online. 2015;20:26150.

17. Burke EJ, Hultgren PB. Will physicians of the future be able to prescribe exercise? J Med Educ. 1975;50(6):624-6.

18. Connaughton AV, Weiler RM, Connaughton DP. Graduating medical students' exercise prescription competence as perceived by deans and directors of medical education in the United States: implications for Healthy People 2010. Public Health Rep. 2001;116(3):226-34.

19. Education CoNiM. Nutrition Education in U.S. Medical Schools. Washington (DC)1985. Available from: http://www. nap.edu/catalog/597.html. [consultado 6 de Junio 2019]

20. Torabi MR, Tao R, Jay SJ, Olcott C. A cross-sectional survey on the inclusion of tobacco prevention/cessation, nutrition/ diet, and exercise physiology/fitness education in medical school curricula. J Natl Med Assoc. 2011;103(5):400-6.

21. Kohlmeier M, Uhley V, Nelson J. Teaching Population Health: Innovative Medical School Curricula on Nutrition 2016 [Available from: https://www.aamc.org/initiatives/diversity/portfolios/449534/nutritionwebinar.html. [consultado 6 de Junio 2019]

22. Kris-Etherton PM, Akabas SR, Bales CW, Bistrian B, Braun L, Edwards MS, et al. The need to advance nutrition education in the training of health care professionals and recommended research to evaluate implementation and effectiveness. Am J Clin Nutr. 2014;99(5 Suppl):1153S-66S.

23. Investments that Work for Physical Activity. British Journal of Sports Medicine. 2012;46(10):709-12.

24. Health Program Nutrition and Physical Activity Initiative. Lots to Lose: How America's Health and Obesity Crisis Threatens our Economic Future. Washington, DC 200052012.

25. Program H, Initiative NaPA. Teaching Nutrition and Physical Activity in Medical School:Training Doctors for PreventionOriented Care. Washington, DC 200052014.
26. Polak R, Pojednic RM, Phillips EM. Lifestyle Medicine Education. Am J Lifestyle Med. 2015;9(5):361-7.

27. National Physical Activity Plan Alliance. National Physical Activity Plan 2013. Available from: http://physicalactivityplan.org/docs/2016NPAP_Finalforwebsite.pdf [consutado 6 de junio 2019].

28. Gates AB, Ritchie IK, Moffatt F, Breda J. Leadership in physical activity: is this the currency of change in the student healthcare curriculum? Br J Sports Med. 2018;52(23):1484-5.

29. Lobelo F, Duperly J, Frank E. Physical activity habits of doctors and medical students influence their counselling practices. Br J Sports Med. 2009;43(2):89-92.

30. Tovar G, López G, Ibáñez M, Alvarado R, Lobelo F, Duperly J. Institutionalized physical activity curriculum benefits of medical students in Colombia. Educ Health (Abingdon). 2016;29(3):203-9.

31. Tovar G, Rodríguez Ä, García G, Tovar J. Actividad física y consejería en estudiantes universitarios de primero y quinto año de medicina de Bogotá, Colombia. Revista Universidad Salud. 2015;18(1):16-23.

32. Initiative HPNaPA. Teaching Nutrition and Physical Activity in Medical School: Training Doctors for Prevention - Oriented Care. Washington, DC 200052014.

33. Ministerio de Salud y Protección Social. Plan Decenal de Salud Pública 2013.

34. Duperly J, Lobelo F, Segura C, Sarmiento F, Herrera D, Sarmiento OL, et al. The association between Colombian medical students' healthy personal habits and a positive attitude toward preventive counseling: cross-sectional analyses. BMC Public Health. 2009;9:218.

35. Frank E, Tong E, Lobelo F, Carrera J, Duperly J. Physical activity levels and counseling practices of U.S. medical students. Med Sci Sports Exerc. 2008;40(3):413-21.

36. Quintero GA. Medical education and the healthcare system-why does the curriculum need to be reformed? BMC Med. 2014;12:213.

37. Lloyd-Jones DM, Hong Y, Labarthe D, Mozaffarian D, Appel LJ, Van Horn L, et al. Defining and setting national goals for cardiovascular health promotion and disease reduction: the American Heart Association's strategic Impact Goal through 2020 and beyond. Circulation. 2010;121(4):586-613.

38. Lobelo F, Rohm Young D, Sallis R, Garber MD, Billinger SA, Duperly J, et al. Routine Assessment and Promotion of Physical Activity in Healthcare Settings: A Scientific Statement From the American Heart Association. Circulation. 2018;137(18):e495-e522. 